\title{
Long common bile duct stricture: Wait, are we on the right track?
}

\author{
Ashish Kumar Jha • Mahesh Kumar Goenka • \\ Ragesh Babu Thandassery
}

Published online: 21 August 2013

(C) Indian Society of Gastroenterology 2013

Editor,

Perforations during endoscopic retrograde cholangiopancreatography (ERCP) are rare. Risk factors include sphincterotomy, sphincter of Oddi dysfunction (SOD), dilated common bile duct (CBD), procedure duration, and stricture dilatation [1]. CBD perforation is very rare, and usually the lower part is involved [2]. We highlight difficulties in recognition through a case we encountered recently. A 67-year-old male with a past history of biliary pancreatitis and cholecystectomy presented with recurrent abdominal pain. Investigations revealed elevated serum alkaline phosphatase and a dilated (diameter $13 \mathrm{~mm}$ ) CBD. At ERCP, cannulation was difficult and took $17.5 \mathrm{~min}$. There was resistance at the lower CBD which disappeared, and the guide wire appeared to have reached the hepatic duct. However, there was poor filling of the bile duct with contrast (Fig. 1a), which was initially thought to be due to a long biliary stricture. Sphincterotomy and plastic stenting were done. However, blood was seen coming through the stent lumen as well as by the side of the stent. In view of suspected vascular injury, the stent was removed, and the bleeding stopped. Repeat cannulation was performed, and this time, adequate contrast filling was achieved. The cholangiogram revealed a dilated and slightly tortuous CBD (Fig. 1b). We assumed SOD, and sphincterotomy was completed followed by stenting. Bile flow was achieved. No postprocedural complications were noted. Retrospectively, when we compared the course of the guide wires (arrow in Fig. 1a, b), it appears that in the initial attempt, the CBD wall was dissected, and the contrast was tracking along the CBD wall. With the self-limiting bleed seen after initial cannulation

\footnotetext{
A. K. Jha $(\bowtie) \cdot M$. K. Goenka $\cdot$ R. B. Thandassery

Institute of Gastroscience, Apollo Gleneagles Hospital, 58 Canal Circular Road, Kolkata 700 054, India

e-mail: ashishjhabn@yahoo.co.in
}

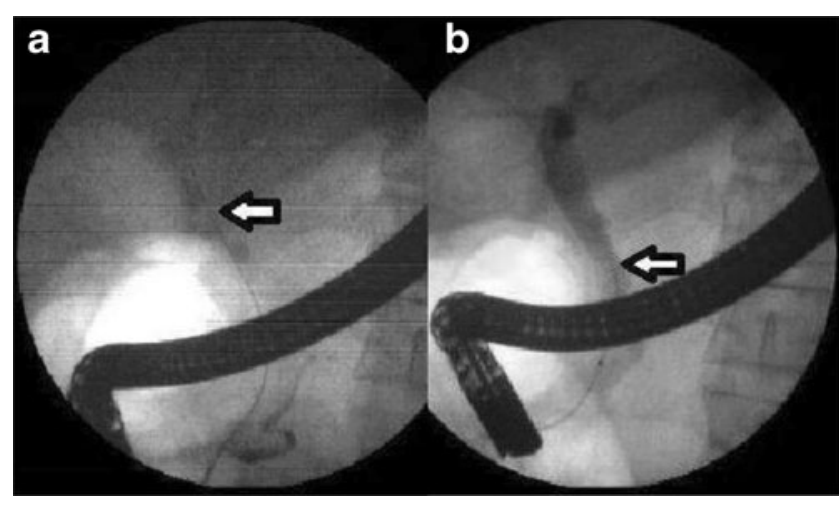

Fig. 1 a Cholangiogram showing poor filling of the bile duct with contrast (the arrow depicts the course of the guide wire). b Cholangiogram showing adequate filling of the bile duct with contrast (the arrow depicts the course of the guide wire)

attempt and absence of rapid washout of contrast, puncture of vascular structure is unlikely. Unfortunately, CT scan or MR imaging was not performed to confirm the dissection of the $\mathrm{CBD}$ wall. We conclude that $\mathrm{CBD}$ wall dissection along its length may occur during cannulation, giving a false appearance of CBD stricture on the cholangiogram. One should consider such a possibility when there is poor filling of the CBD lumen on the cholangiogram, especially after a prolonged and difficult cannulation attempt.

\section{References}

1. Enns R, Eloubeidi MA, Mergener K, et al. ERCP-related perforations: risk factors and management. Endoscopy. 2002;34:293-8.

2. Lee YS, Moon JH, Ko BM, et al. Endoscopic closure of a distal common bile duct perforation caused by papillary dilation with a large-diameter balloon (with video). Gastrointest Endosc. 2010; 72:616-8. 